29. Cornell, S. J., Isham, V. S., Smith, G. \& Grenfell, B. T. Spatial parasite transmission, drug resistance, and the spread of rare genes. Proc. Natl Acad. Sci. USA 100, 7401-7405 (2003).

30. Dobson, A. P. \& Roberts, M. The population dynamics of parasitic helminth communities. Parasitology 109, S97-S108 (1994).

Supplementary Information accompanies the paper on www.nature.com/nature.

Acknowledgements This work was undertaken at the University of Stirling and we thank the Natural Environment Research Council for funding the project. We also thank R. Norman of the University of Stirling for modelling assistance.

Competing interests statement The authors declare that they have no competing financial interests.

Correspondence and requests for materials should be addressed to J.L. (Joanne.Lello@csiro.au).

\section{Tug-of-war over reproduction in a social bee}

\section{Philipp Langer $^{1}$, Katja Hogendoorn ${ }^{2} \star \&$ Laurent Keller $^{1}$}

${ }^{1}$ Department of Ecology and Evolution, University of Lausanne, Biology Building, 1015 Lausanne, Switzerland

${ }^{2}$ School of Biological Sciences, Flinders University of South Australia, Adelaide SA 5001, Australia

* Present address: Centre for Plant and Pest Management, Adelaide University, Glen Osmond SA 5064, Australia

One of the main transitions in evolution is the shift from solitary organisms to societies with reproductive division of labour ${ }^{1,2}$. Understanding social evolution requires us to determine how ecological, social and genetic factors jointly influence group stability and partitioning of reproduction between group members $^{3-8}$. Here we test the role of the three key factors predicted to influence social evolution by experimentally manipulating them in a social allodapine bee. We show that increased relatedness between nestmates results in more even reproduction among group members and a greater productivity per individual. By contrast, the degree of reproductive skew is not influenced by the opportunity for solitary breeding or by the potential benefits of cooperation. Relatedness also has a positive effect on group stability and overall productivity. These findings are in line with predictions of the tug-of-war models, in which the degree of reproductive division of labour is determined primarily by selfish competition between group members. The alternative view, where the degree of reproductive skew is the outcome of a social contract between potential breeders, was not supported by the data.

Reproductive skew models can be divided into two classes on the basis of the assumed power relations in a social group. In transactional models, group members yield reproduction to each other in return for specific benefits, whereas in tug-of-war models reproductive sharing reflects each group member's inability to effectively monopolize reproduction $^{5,6}$ (Table 1). The first of the two main transactional models, the concession model, assumes that a dominant individual fully controls both group membership and the fraction of total group reproduction that a subordinate obtains ${ }^{9-11}$. Under this model, the dominant yields just enough reproduction to a subordinate to make it worthwhile for the subordinate to stay and cooperate peacefully rather than leave the group to reproduce solitarily. This model predicts that reproductive skew increases with higher genetic relatedness between the dominant and the subordinate $(r)$, and higher overall reproductive output of a group of two breeders relative to the output of a single dominant $(k)$, but decreases with higher expected solitary reproduction of a potential subordinate relative to that of a lone dominant $(x$, lower values of $x$ indicating harsher ecological constraints on solitary breeding). This is because the subordinate gains greater fitness benefits from cooperation with increased $r$ and $k$, and thus requires less direct reproduction. However, when the chances of successful solitary breeding of the subordinate are high (increased $x$ ), the dominant has to concede a larger fraction of colony reproduction to the subordinate to make staying in the group profitable for the latter. The other main transactional model, the restraint model, assumes that the dominant controls group membership but that a subordinate fully controls its reproductive share within the group ${ }^{12}$. Under this model, the subordinate captures the largest share of reproduction that the dominant will tolerate before ejecting the subordinate, resulting in opposite predictions to the concession model (Table 1). Finally, tug-of-war models assume that both dominant and subordinate individuals have only limited control over the allocation of reproduction and must expend effort to increase their shares of the total group output. The main prediction of this model is that reproductive skew is negatively correlated with or independent of relatedness because struggle over reproduction takes place at the cost of overall group productivity, with the effect that higher relatedness acts as a break on the investment in reproductive competition ${ }^{13}$. This model predicts that skew is not correlated with group productivity $(k)$ or the ecological constraint on solitary breeding $(x)$. Unlike the two transactional models, where relatedness has no influence on group productivity, the tug-of-war model predicts a positive correlation between breeder relatedness and total group output, because related females allocate less energy to reproductive competition.

We tested the predictions of reproductive skew models in the Australian allodapine bee Exoneura nigrescens by experimentally manipulating all three parameters $(r, x$ and $k)$. Several characteristics make this species an ideal experimental system. First, cooperation is facultative, with females nesting either solitarily or with up to three other females ${ }^{14}$, which allows direct comparison between social and solitary productivities $(k)$. Second, there are no morphological castes, hence all females have the potential to reproduce $^{11}$. Third, a high proportion $(51 \%)$ of multi-female nests contain two females ${ }^{15}$, the type of groups for which most reproductive skew models have been developed. Fourth, withinnest relatedness $(r)$ is variable $\mathrm{e}^{14,16,17}$ and can be experimentally manipulated. Fifth, nests are built primarily in dead dried flower stalks of grasstrees (Xanthorrhoea minor) and Melaleuca squarrosa ${ }^{15}$,

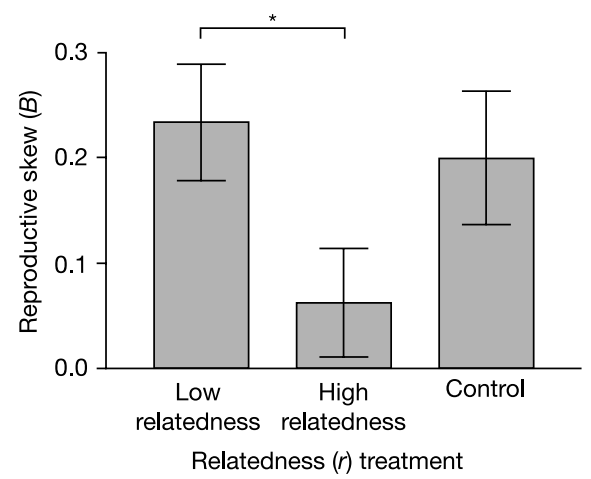

Figure 1 Reproductive skew in nests of the high- and low-relatedness treatment, and in unmanipulated control nests. Overall skew was significantly greater than zero in both the low-relatedness treatment (one-sample $t$-test, $t=4.2$, d.f. $=6, P=0.005$ ) and natural nests $(t=3.2$, d.f. $=8, P=0.01)$. In the high-relatedness treatment, however, skew was not significantly different from zero $(t=1.2$, d.f. $=12, P=0.24)$, the expected value if breeders share reproduction equally ${ }^{29}$. Error bars indicate standard errors, $n=29$. 


\begin{tabular}{|c|c|c|c|c|}
\hline Association & Concession models & Restraint model & Tug-of-war model & Experimental data \\
\hline Skew versus relatedness $(r)$ & Positive & Negative & None or negative & Negative \\
\hline Skew versus subordinate's solitary nesting success $(x)$ & Negative & Positive & None & None \\
\hline Skew versus productivity benefits of cooperative nesting $(k)$ & Positive & Negative & None & None \\
\hline Relatedness $(r)$ versus overall group productivity & None & None & Positive & Positive \\
\hline
\end{tabular}

The effect of each parameter assumes that the other parameters and group size are held constant.

providing opportunities for controlling nesting site abundance and thus $x$, the constraints on solitary breeding. Sixth, because nesting site abundance varies greatly in the wild ${ }^{17,18}$, individuals are likely to be responsive to experimental manipulation of nesting opportunities. Finally, whole colonies can be easily transferred to experimental plots that differ in specific resources, allowing the manipulation of $k$, the relative productivity of social versus solitary nests.

To test the role of relatedness $(r)$ on reproductive skew, nest cofounding by related bees was favoured in a high-relatedness treatment but restrained in a low-relatedness treatment (see Methods). The treatment proved successful, resulting in a significantly greater relatedness among foundresses in the high-relatedness treatment (mean $r \pm$ s.e.m. $=0.51 \pm 0.08$ ) compared with the low-relatedness treatment $(r=0.08 \pm 0.08$; unpaired $t$-test, $t=3.60$, d.f. $=19, P<0.002)$, whereas unmanipulated control nests showed an intermediate value $(r=0.38 \pm 0.11)$. Relatedness had a significant effect on the degree of reproductive skew, with significantly higher skew (unpaired $t$-test, $t=2.11$, d.f. $=18$, $P<0.05)$ in the low-relatedness compared with the high-relatedness treatment (Fig. 1). The influence of relatedness on skew was further demonstrated by a significant negative correlation between skew and relatedness across all colonies (Pearson's $r=-0.53$, $n=29, P<0.005$ ) (Fig. 2). Variation in relatedness also affected overall group productivity: two-female nests in the high-relatedness treatment $(7.2 \pm 0.9$ offspring) were significantly more productive (unpaired $t$-test, $t=2.31$, d.f. $=17, P=0.03$ ) than two-female nests in the low-relatedness treatment ( $4.0 \pm 0.4$ offspring). Across all colonies, the productivity per female was also positively correlated with breeder relatedness (Pearson's $r=0.35, n=32$, $P<0.05)$.

To test the influence of solitary breeding success $(x)$ on reproductive skew, we manipulated the availability of nesting sites. In a low-nest-availability treatment we removed all nesting substrates (dead stalks of grasstrees), whereas in a high-nest-availability treatment we added supplementary nesting sites. Our treatment was clearly effective, because females in the low-nest-availability treatment, lacking alternative nesting substrate, remained in their initial nest in a significantly higher proportion (26 out of 66 nests) than did those in the high-nest-availability treatment ( 11 out of 78 nests; Fisher's exact test, $P=0.001)$. However, nest availability had

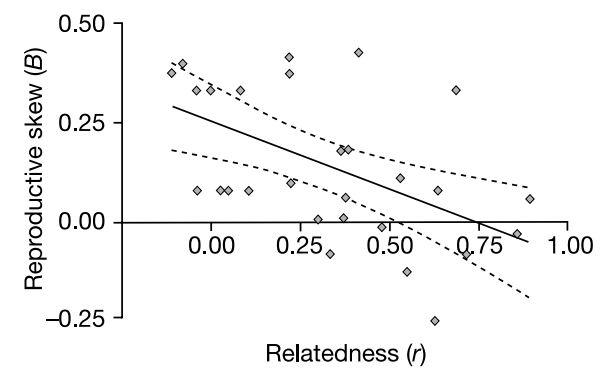

Figure 2 Correlation between relatedness and reproductive skew across all nests. The data include unmanipulated natural nests as well as high-relatedness and lowrelatedness treatment nests. The straight line indicates the linear regression with its 95\% confidence interval (dotted curve), slope $-0.34 \pm 0.10, n=29$. no significant effect on reproductive skew (unpaired $t$-test, $t=0.39$, d.f. $=14, P=0.70)$ (Fig. 3).

To test the influence of $k$, the relative productivity of two- versus one-female nests, we placed occupied nests in plots with rich and poor floral resources, because this has been shown to affect the relative benefits of group living in this species ${ }^{19}$. Our treatment was successful given that two-female nests were disproportionately more productive than single-female nests in rich floral plots $(k=3.4)$, but not in poor floral plots where single- and two-female nests showed similar productivities $(k=1.1)$. Consequently, a twoway ANOVA on productivity revealed a significant interaction between the presence of one versus two females per nest and the level of floral resources $\left(F=10.1\right.$, d.f. $\left.{ }_{n, d}=1,88, P=0.002\right)$; the number of females per nest $\left(F=12.5\right.$, d.f. $\left.{ }_{n, d}=1,88, P<0.001\right)$ and the level of floral resources $\left(F=10.4\right.$, d.f. $\left._{n, d}=1,88, P<0.002\right)$ also had a significant effect on productivity. Despite the important difference in $k$ between rich and poor floral plots, there was no significant difference in skew (unpaired $t$-test, $t=0.86$, d.f. $=22$, $P=0.40$ ) (Fig. 4). It is unlikely that the lack of difference in skew can be explained by the inability of females to distinguish between the two environments, because the bees founded significantly more joint colonies in rich floral plots, the environment providing larger rewards for cooperation (20 multi-female and 22 single-female nests in rich floral plots versus 14 multi-female and 47 single-female nests in poor floral plots; two-sided Fisher's exact test, $P=0.01$ ).

The results of this study do not support the predictions of the two transactional models (Table 1). None of the four predictions of the concession model was found to hold in E. nigrescens. Similarly, three predictions of the restraint model were not supported by our data. There was no significant positive relationship between reproductive skew and the constraints on solitary breeding $(x)$, and no significant negative relationship between skew and the relative productivity of two- versus one-female nests $(k)$. Importantly, the lack of significant association between skew and relative productivity is unlikely to result from a lack of power of the test, because the restraint model would predict complete skew in the rich floral plots (calculated from equation (2) in ref. 12 using the observed parameters, $k=3.4$ and $r=0.52 \pm 0.08$, and all possible values of $e$, the cost of eviction, and $x$ ), whereas skew was far from complete in the experimental data. Finally, further evidence against the restraint model comes

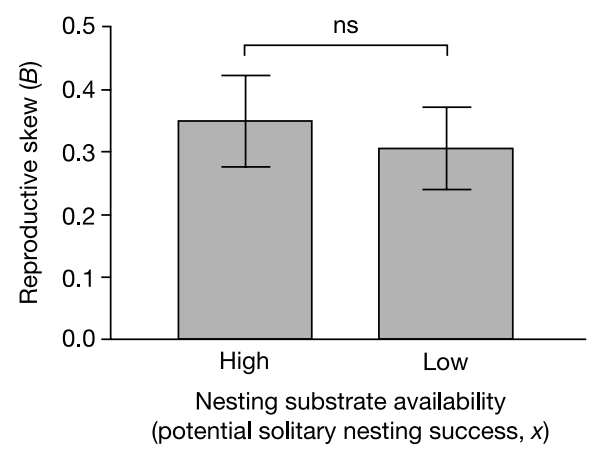

Figure 3 Reproductive skew in treatments with high or low nesting substrate availability. The availability of nesting substrate is a direct measure of a nestmate's potential solitary nesting success $(x)$. Error bars indicate standard errors, $n=16$. 


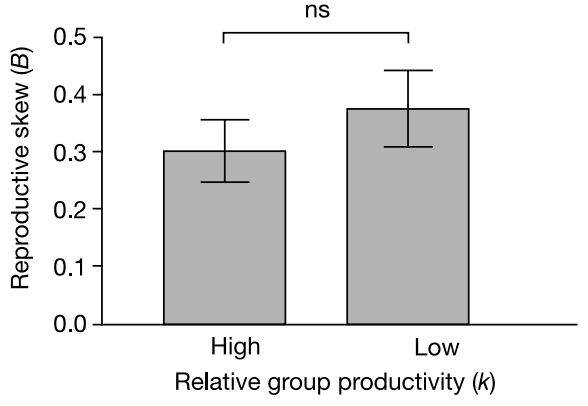

Figure 4 Reproductive skew in treatments with rich and poor floral resources (high and low $k$, respectively). Error bars indicate standard errors, $n=24$.

from the finding that group productivity was positively associated with relatedness, whereas this model predicts no association.

By contrast, the results matched each of the four predictions of the tug-of-war models. Reproductive skew was negatively associated with the relatedness between breeders $(r)$ and independent of both the constraints on solitary breeding $(x)$ and the productivity benefits of cooperative nesting $(k)$. Moreover, overall group productivity was positively associated with $r$, as predicted by tug-of-war models. This association, which has previously been reported in some other social insects ${ }^{20}$, is expected from the lower investments in reproductive competition in groups where individuals are more related because of kin benefits. Finally, we also found that the tendency of females to join another female was significantly influenced by their relatedness: in the high-relatedness treatment, where females could readily associate with related individuals, the bees founded 33 multi-female and 30 single-female nests, versus 5 multifemale and 52 single-female nests in the low-relatedness treatment (two-sided Fisher's exact test, $P<0.0001$ ). This result shows that females can assess relatedness and prefer to breed alone rather than with unrelated individuals, despite much lower survival rates of solitary nests in this species ${ }^{21}$. This is in line with tug-of-war models that predict higher productivity and lower competition in nests of related breeders, but is incompatible with transactional models that predict that females should join independently of relatedness ${ }^{11,22}$.

This study is the first to succeed in experimentally manipulating relatedness between breeders in a natural environment. Importantly, our data show that Exoneura females can assess each of the three parameters involved in reproductive skew models $(r, x$ and $k$ ) and actively adapt their reproductive strategy accordingly, a basic yet never tested assumption of skew models ${ }^{23}$. The overall results strongly support the tug-of-war model, in which a dominant is unable to fully control the allocation of reproduction and group membership in an animal society ${ }^{13,24}$, and partitioning of reproduction thus results from selfish and costly efforts of individuals to secure the greatest possible share of the group's output. Accordingly, removals of brood and mutual egg eating have both been observed in nests of this species ${ }^{19}$. Inversely, there was no support for the alternative view that partitioning of reproduction ultimately reflects a 'social contract' between group members. More generally, this study emphasizes the relative complexity of insects' behavioural repertoire and their ability to adaptively modulate their level of competitive effort according to social attributes such as the relatedness of other group members.

\section{Methods}

\section{Manipulation of relatedness $(\boldsymbol{r})$}

Four hundred nests were collected in March 1999 at Cobboboonee State Forest, Victoria, Australia. To construct high-relatedness nests, we placed ten nestmate females (related ${ }^{14}$ ) from overwintered nests into each of 30 vials deposited in two $30 \times 30 \mathrm{~m}$ experimental plots. Within half a metre around each vial, we placed ten stalks of nesting substrate (dry grasstree flower scapes $)^{18}$ to provide females with opportunities to initiate a nest with related individuals ${ }^{15,17}$. To construct low-relatedness nests, we followed the same procedure but placed ten females from ten different nests in each of 30 vials, which decreased the opportunity for females to initiate a colony with a related female. As for the high-relatedness treatment, the vials were placed in two experimental plots. All nests were collected in June after the founding period and X-rayed to determine nest contents. The multi-female nests were immediately returned to a single field site to provide a similar environment and thus control other ecological parameters possibly influencing skew. Nests were collected in January 2000 after brood production. The experiment was repeated with another 400 nests the following year, swapping the plots used for high- and lowrelatedness treatments. As $t$-tests showed no significant effects of year and plot on productivity, within-nest relatedness or reproductive skew, we pooled the data and present the combined results.

Maternity and relatedness within nests were estimated using microsatellites and dissection data (see below). Three out of 33 nests contained a mother and her daughter as breeders. They were excluded because of the potential effect of matrifilial structure on skew $^{13,25}$.

\section{Manipulation of ecological constraints on solitary nest founding $(x)$}

In January 2000, we removed all existing nests and nesting substrate in four similar plots of $50 \times 50 \mathrm{~m}$, and deposited approximately 40 established nests from a nearby population per plot. In two plots (high-nest-availability treatment), we placed ten stalks of empty nesting substrate nearby each deposited nest, providing ample possibilities for solitary nesting ${ }^{18}$. In the other two plots (low-nest-availability treatment), no nesting substrate was provided, resulting in harsh ecological constraints on solitary nesting. All nests were collected in December 2000 for genetic analysis. Because pairs of plots within each treatment showed no significant differences in productivity, within-nest relatedness and reproductive skew ( $t$-tests, all nonsignificant), data were combined.

\section{Manipulation of relative group productivity $(k)$}

A previous study showed that the productivity of multi-female colonies relative to singlefemale colonies $(k)$ is lower in poor-resource than in high-resource areas ${ }^{19}$. To determine the effect of $k$ on skew, we selected three pairs of plots $(50 \times 50 \mathrm{~m}$ each $)$, one with high and the other with low flower abundance. None of the plots originally contained nesting substrates or occupied nests. In July 2000, we placed about 50 newly founded nests in each plot. We collected all nests in December 2000 and assessed productivity of single- and multi-female nests as well as relatedness, maternity and skew. In each plot, the relative productivity of two- versus single-female nests $(k)$ was calculated by dividing the mean number of offspring in two-female nests by the mean number of offspring in single-female nests ${ }^{6}$. The number of offspring did not differ significantly between plots of the same treatment in single-female nests ( $t$-tests, all nonsignificant). Similarly, there was no significant difference in productivity between plots in two-female nests ( $t$-tests, all nonsignificant), and we therefore combined the plots of the same treatment. Two nests containing a breeding mother-daughter association were excluded from the analyses.

\section{Genetic analyses}

All adults, brood and eggs were genotyped at five highly polymorphic microsatellite loci (N22, N60, N81, N83, R74; ref. 26). Adults were furthermore dissected, and the sizes of oocytes, insemination status, yellow body content and wing wear recorded ${ }^{27}$. The genetic and morphological data allowed us to unambiguously distinguish between the

foundresses and their daughters, and to determine maternity of all offspring. Sample sizes were 74 females and 335 offspring, 41 females and 220 offspring, and 58 females and 201 offspring for the first, second and third experiment, respectively.

Relatedness between foundresses was calculated using the program Relatedness 5.0.8 (ref. 28). Allele frequencies were bias-corrected at the nest level because nestmates are related, and nests were weighted equally.

\section{Reproductive skew}

The degree of reproductive skew was quantified with the $B$ index, where zero indicates random distribution of reproduction among group members, positive values a higher skew and negative values a lower skew than expected by chance ${ }^{29}$. We quantified skew for overall offspring production because of the low number of males produced. Separate analyses for each sex gave similar qualitative results, as there was no trade-off among breeders for male and female production ${ }^{30}$. Of the multi-female nests considered in this study, 63 nests contained two females and 20 nests three or more females. Statistical analyses considering only two-female nests or all nests with multiple females yielded identical conclusions. We therefore present only the analyses comprising all nests.

Received 4 December 2003; accepted 23 February 2004; doi:10.1038/nature02431.

1. Maynard Smith, J. \& Szathmáry, E. The Major Transitions in Evolution (W. H. Freeman, Oxford, 1995).

2. Keller, L. (ed.) Levels of Selection in Evolution (Princeton Univ. Press, Princeton, 1999).

3. Keller, L. \& Reeve, H. K. Partitioning of reproduction in animal societies. Trends Ecol. Evol. 9, 98-102 (1994).

4. Field, J., Solis, C. R., Queller, D. C. \& Strassmann, J. E. Social and genetic structure of paper wasp cofoundress associations: tests of reproductive skew models. Am. Nat. 151, 545-563 (1998).

5. Johnstone, R. A. Models of reproductive skew: a review and synthesis. Ethology 106, 5-26 (2000).

6. Reeve, H. K. \& Keller, L. Tests of reproductive-skew models in social insects. Annu. Rev. Entomol. 46, 347-385 (2001).

7. Clutton-Brock, T. H. et al. Cooperation, control, and concession in meerkat groups. Science 291, 478-481 (2001).

8. Haydock, J. \& Koenig, W. D. Reproductive skew in the polygynandrous acorn woodpecker. Proc. Nat Acad. Sci. USA 99, 7178-7183 (2002).

9. Vehrencamp, S. L. A model for the evolution of despotic versus egalitarian societies. Anim. Behav. 31, 667-682 (1983).

10. Emlen, S. T. The evolution of helping. I. An ecological constraints model. Am. Nat. 119, 29-39 (1982). 
11. Reeve, H. K. \& Ratnieks, F. L. W. in Queen Number and Sociality in Insects (ed. Keller, L.) 45-85 (Oxford Univ. Press, Oxford, 1993).

12. Johnstone, R. A. \& Cant, M. A. Reproductive skew and the threat of eviction: a new perspective. Proc R. Soc. Lond. B 266, 275-279 (1999).

13. Reeve, H. K., Emlen, S. T. \& Keller, L. Reproductive sharing in animal societies: reproductive incentives or incomplete control by dominant breeders? Behav. Ecol. 9, 267-278 (1998).

14. Schwarz, M. P., Bull, N. J. \& Hogendoorn, K. Evolution of sociality in the allodapine bees: a review of sex allocation, ecology and evolution. Insectes Soc. 45, 349-368 (1998).

15. Silberbauer, L. X. \& Schwarz, M. P. Life-cycle and social-behavior in a heathland population of the allodapine bee, Exoneura bicolor (Hymenoptera, Apidae). Insectes Soc. 42, 201-218 (1995).

16. Schwarz, M. P. Female-biased sex-ratios in a facultatively social bee and their implications for social evolution. Evolution 48, 1684-1697 (1994).

17. Silberbauer, L. X. Founding patterns of Exoneura bicolor in Cobboboonee State Forest, S.W. Victoria. Aust. Zool. 28, 67-73 (1992)

18. Bull, N. J. \& Schwarz, M. P. The habitat saturation hypothesis and sociality in an allodapine bee: cooperative nesting is not 'making the best of a bad situation'. Behav. Ecol. Sociobiol. 39, 267-274 (1996).

19. Stevens, M. I. Egg Stock Piling Strategies and Benefits to Group Living in Exoneura bicolor Smith (Apidae, Xylocopinae) Thesis, Flinders Univ. South Australia, Adelaide (1997)

20. Gadagkar, R., Chandrashekara, K., Chandran, S. \& Bhagavan, S. Queen success is correlated with worker-brood genetic relatedness in a primitively eusocial wasp (Ropalidia marginata). Experientia 49, 714-717 (1993).

21. Hogendoorn, K. \& Zammit, J. Benefits of cooperative breeding through increased colony survival in an allodapine bee. Insectes Soc. 48, 392-397 (2001)

22. Reeve, H. K. in The Social Biology of Wasps (eds Ross, K. G. \& Matthews, R. W.) 99-148 (Cornell Univ. Press, Ithaca, 1991)

23. Kokko, H. Are reproductive skew models evolutionarily stable? Proc. R. Soc. Lond. B 270, 265-270 (2003).

24. Clutton-Brock, T. H. Reproductive skew, concessions and limited control. Trends Ecol. Evol. 13, 288-292 (1998).

25. Reeve, H. K. \& Keller, L. Partitioning of reproduction in mother-daughter versus sibling associations-a test of optimal skew theory. Am. Nat. 145, 119-132 (1995).

26. Langer, P., Molbo, D. \& Keller, L. Polymorphic microsatellite loci in Allodapine bees for investigating the evolution of social behaviour. Mol. Ecol. Notes (in the press).

27. Schwarz, M. P. Persistent multi-female nests in an Australian Allodapine bee, Exoneura bicolor (Hymenoptera, Anthophoridae). Insectes Soc. 33, 258-277 (1986).

28. Queller, D. C. \& Goodnight, K. F. Estimating relatedness using genetic markers. Evolution 43, 258-275 (1989)

29. Nonacs, P. Measuring and using skew in the study of social behavior and evolution. Am. Nat. 156, 577-589 (2000)

30. Fournier, D. \& Keller, L. Partitioning of reproduction among queens in the Argentine ant, Linepithema humile. Anim. Behav. 62, 1039-1045 (2001).

Supplementary Information accompanies the paper on www. nature.com/nature.

Acknowledgements We thank C. Roger, P. Jordan and G. Deppierraz for help in genotyping N. DiMarco for extractions, M. P. Schwarz for methodological advice, A. Marazzi for statistica assistance, J. Zammit, C. van der Muren, E. Geertsema and T. Taraldsrud for help in setting up field experiments, and R. Hammond, M. Chapuisat, P. Christe, S. Helms Cahan, K. Parker

D. Queller and F. Ratnieks for comments on the manuscript.

Competing interests statement The authors declare that they have no competing financia interests.

Correspondence and requests for materials should be addressed to P.L.

(Philipp.Langer@ie-zea.unil.ch) or L.K. (Laurent.Keller@ie-zea.unil.ch).

\section{Genetic changes associated with floral adaptation restrict future evolutionary potential}

\section{Rebecca A. Zufall ${ }^{1,2}$ \& Mark D. Rausher ${ }^{1}$}

${ }^{1}$ Department of Biology, Box 90338, Duke University, Durham, North Carolina 27708, USA

${ }^{2}$ Current address: Department of Biological Sciences, Smith College, Northampton, Massachusetts 01063, USA

A commonly accepted evolutionary principle is that adaptive change constrains the potential directions of future evolutionary change $^{1-3}$. One manifestation of this is Dollo's law, which states that character elimination is irreversible ${ }^{4,5}$. Although the common occurrence of irreversibility has been documented by phylogenetic analyses of phenotypic transitions, little is known about the underlying causes of this phenomenon ${ }^{4}$. One explanation for evolutionary irreversibility relies on the fact that many characteristics result from interactions between multiple gene products $^{4,6}$. Such characteristics may often be eliminated by inactivation of just one gene in the network. If they serve no other functions, other genes of the network are then free to accumulate mutations or evolve new functions. Evolutionary change after character loss results in the accumulation of redundant loss-of-function mutations. Such pathway degeneration makes it very unlikely that the characteristic will re-evolve, because multiple simultaneous mutations would be required ${ }^{4}$. Here we describe what appear to be the initial stages of such degeneration in the anthyocyanin pigment pathway associated with an adaptive change from blue to red flowers in the morning glory Ipomoea quamoclit.

The ancestral floral colour in the genus Ipomoea is blue/purple $e^{7,8}$ (Fig. 1a, b). A number of independent transitions to other colours have occurred in this genus ${ }^{7-9}$. One such transition is represented by a small, well-supported clade ${ }^{10}$ of red-flowered species in the section Mina, subgenus Quamoclit, including I. quamoclit ${ }^{7}$ (Fig. 1a, b), which are pollinated primarily by hummingbirds $s^{8,11,12}$. Unlike typical blue-flowered Ipomoea species, which are pollinated primarily by bees and exhibit a set of characteristics associated with adaptation to bee pollination (for example, a broad floral tube, small quantities of nectar, inserted stigma and anthers and nonversatile anthers) ${ }^{8,13}$, Mina species exhibit a suite of floral traits, including red pigmentation, typically associated with bird pollination (for example, narrow tube, copious nectar production, exserted stigma and anthers and versatile anthers $)^{7,8,13}$. The transition to red flowers in this group appears to represent an adaptive shift to association with a different type of pollinator.

Floral colour in Ipomoea is determined largely by the type of anthocyanin pigment produced. Pigments derived from cyanidin typically produce blue/purple flowers, whereas pigments derived from pelargonidin typically produce red flowers ${ }^{14-16}$. Cyanidin differs from pelargonidin by possessing an extra hydroxyl group, which is added by the enzyme flavonoid $3^{\prime}$-hydroxylase $\left(F 3^{\prime} \mathrm{H}\right)^{16}$. This enzyme creates a branch in the biosynthetic pathway, with the enzymes downstream of $\mathrm{F}^{\prime} \mathrm{H}$ (that is, dihydroflavonol reductase (DFR), anthocyanidin synthase (ANS) and UDP glucose flavonoid 3-glucosyltransferase (UF3GT)) participating in both branches (Fig. 2). In I. nil, I. purpurea and I. tricolor, virtually all of the flux normally flows down the cyanidin branch of the pathway, producing blue flowers. However, mutations in these species that block the cyanidin branch produce red pigments and flowers (Fig. 1c), indicating that these downstream enzymes are substrate generalists capable of metabolizing both the hydroxylated and nonhydroxylated intermediates ${ }^{16,17}$.

Thin-layer chromatography of anthocyanidins from several Mina species reveals that the red floral pigments of these species are derived from pelargonidin (Fig. 1b, c). The evolutionary transition to red flowers in this group was thus brought about by inactivation of the cyanidin branch, which can be accomplished in two ways, either by inactivation of $\mathrm{F}^{\prime} \mathrm{H}$ or by the evolution of substrate specificity-loss of the ability to metabolize hydroxylated substrates-by one of the downstream enzymes, DFR or ANS. Substrate specificity of DFR has been demonstrated in several unrelated genera, including Petunia ${ }^{18}$ and Arabidopsis ${ }^{19}$. Here we show that both of these types of change have occurred in the lineage leading to I. quamoclit.

The single-copy $\mathrm{F3}^{\prime} \mathrm{h}$ gene from $I$. quamoclit exhibits a high sequence similarity to its homologue from $I$. purpurea. The two sequences exhibit no indels and are $94.7 \%$ similar at the nucleotide level and $93.5 \%$ similar at the amino-acid level. Although no obvious features (for example, premature stop codons or frame shifts) indicate that the gene is non-functional, our experiments 